\title{
Poligrafía en niños con enfermedad neuromuscular
}

\author{
DANIEL ZENTENO A.*, IVÁN RODRÍGUEZ N.**, CARLA RIVAS B.***, \\ RUBÉN PEÑA Z.****, IGNACIO MOLINA D.***** y JAIME TAPIA Z.******
}

\section{Polygraphy in children with neuromuscular disease}

Introduction: A high prevalence of sleep-disordered breathing (SDB) has been reported in neuromuscular disease (NMD) patients. Our aim was to describe the results of sleep studies performed by overnight polygraphy in pediatric ward of a public hospital from Concepción, Chile. Additionally, we purposed to define its utility in the treatment of children with NMD. Methods: Records of NMD patients admitted at G. Grant Benavente Hospital, from 2011 to 2015 were considered. The therapeutic approaches were classified as: non invasive ventilation, surgical treatment and follow up. Results: From 36 patients initially admitted in the study 5 were excluded. Patients median age was 10 years-old (range: $0.3-19), 74 \%(n=23)$ were males. Diagnosis were: Duchenne muscular dystrophy in 12 patients (39\%), Myelomeningocele in 6 (19\%), Hypotonic syndrome in 5 (16\%), Miopathy in 3 (10\%), Spinal muscular atrohpy in $3(10 \%)$ and other NMD in 2 patients (6\%). Median of polygraphy valid time was $7.3 \mathrm{~h}$ (range:4.3-10.5). Median of mean values of $\mathrm{O}_{2}$ saturation was $97 \%$ (range: 91-99\%) and median of minimum $\mathrm{O}_{2}$ saturation was $90 \%$ (51-95\%). Six polygraphies (19\%) were normal and 25 (81\%) showed some degree of SDB. From this group $60 \%$ had a mild, $28 \%(n=7)$ had a moderate and $12 \%(n=3)$ presented a severe SDB. Fifteen patients (65\%) were under non invasive ventilation, nine (29\%) of them received medical treatment and two of them (6\%) surgical treatment. There was no difference between the magnitude of SDB and therapeutic approach. Moreover, no association between the severity of $S D B$ and therapeutic approach was found. Conclusion: Polygraphy allows objective diagnosis of SDB in children with NMD and is a suitable tool to define therapeutic conducts.

Key words: Polygraphy; children; neuromuscular disease; Sleep disorders breathing.

\section{Resumen}

Introducción: Los pacientes con enfermedades neuromusculares (ENM) presentan una alta prevalencia de trastornos respiratorios del sueño (TRS). El objetivo de este estudio fue describir los resultados de estudios poligráficos y mostrar su utilidad para el establecimiento de conductas terapéuticas en niños con ENM de un hospital público de Chile. Metodología: Se consideraron registros de $P G$ de niños con ENM. Las conductas terapéuticas fueron clasificadas como: asistencia ventilatoria no invasiva (AVNI), cirugía y observación y seguimiento. Los resultados se expresan en mediana y rango. Los tests de Kruskal-Wallis y $\chi^{2}$ fueron empleados. Fue considerado significativo un $p<0,05$. Resultados: Al estudio ingresan 36 pacientes, siendo excluidos 5, la mediana de edad fue 10 años (0,3-19), 74\% varones. Diagnósticos: Distrofia neuromuscular de Duchenne 39\% $(n=12)$, Mielomeningocele $19 \%(n=6)$, Síndrome hipotónico 16\% $(n=5)$, Miopatía 10\% $(n=3)$, Atrofia espinal $10 \%(n=3)$, otros $6 \%(n=2)$. El tiempo validado de la poligrafía fue $7,3 \mathrm{~h}(4,3-10,5)$, la mediana de la saturación

\footnotetext{
* Pediatra Broncopulmonar. Profesor Asistente, Departamento de Pediatría, Facultad de Medicina, Universidad de Concepción. Hospital Guillermo Grant Benavente, Concepción.

** Kinesiólogo. Escuela de Kinesiología, Facultad de Ciencias de la Salud, Universidad San Sebastián, Concepción, Chile.

*** Enfermera Universitaria. Hospital Guillermo Grant Benavente, Concepción, Chile.

**** Becado de Pediatría. Departamento de Pediatría, Facultad de Medicina, Universidad de Concepción, Chile.

***** Interno de Medicina. Departamento de Pediatría, Facultad de Medicina, Universidad de Concepción, Chile.

****** Pediatra Cardiólogo. Profesor Asistente, Departamento de Pediatría, Facultad de Medicina, Universidad de Concepción. Hospital Guillermo Grant Benavente, Concepción, Chile.
} 
de $\mathrm{O}_{2}$ promedio fue 97\% (91-99) y de la saturación de $\mathrm{O}_{2}$ mínima 90\% (51-95). Las poligrafías fueron normales en 6 pacientes (19\%) y sugerentes de TRS en 25 (81\%). Entre ellas se consideró SAHOS leve $60 \%(n=15)$, moderado $28 \%(n=7)$ y severo $12 \%(n=3)$. En 20 pacientes $(65 \%)$ se decidió iniciar AVNI, en 9 (29\%) observación y seguimiento y en 2 (6\%) tratamiento quirúrgico. No existió asociación entre la categorización de gravedad de SAHOS y conducta terapéutica. Conclusión: La poligrafía permite el diagnóstico objetivo de TRS en niños con ENM y constituye una herramienta útil para determinación de conductas terapéuticas.

Palabras clave: Poligrafia; Niños; Enfermedad neuromuscular; Trastornos respiratorios del sueño.

\section{Introducción}

Los pacientes con enfermedades neuromusculares (ENM) presentan una mayor prevalencia de trastornos respiratorios del sueño (TRS) en comparación a la población pediátrica sana; siendo su inicio variable según el tipo de patología y presentación individual ${ }^{1}$.

Se estima que más del $40 \%$ presentará a lo largo de su vida un TRS, el cual, puede asociarse a alteraciones del intercambio gaseoso, arquitectura del sueño, calidad de vida y morbimortalidad ${ }^{1}$. Desde el punto de vista fisiopatológico, los TRS son generados principalmente por la afección de la musculatura inspiratoria y el aumento de la resistencia de la vía aérea ${ }^{1,2}$.

Si bien los pacientes con ENM pueden presentar TRS en etapas iniciales de la vida, la mayor parte de ellos los manifiestan al evolucionar la enfermedad de base, por lo tanto, su detección precoz es fundamental para realizar un tratamiento oportuno ${ }^{3,4}$.

El examen de elección para el diagnóstico de los TRS es la polisomnografía. No obstante, dado el alto costo de su implementación, han sido desarrollados exámenes alternativos como la poligrafía (PG), la cual, ha mostrado tener un rendimiento diagnóstico aceptable en la población de interés ${ }^{5-7}$.

La ventilación no invasiva (VNI) constituye uno de los pilares de tratamiento de los TRS en pacientes con $\mathrm{ENM}^{8}$. En este contexto, se ha observado que la VNI revierte la hipoxemia, normaliza la arquitectura del sueño, reduce la morbilidad y mejora la calidad de vida en niños con $\mathrm{ENM}^{8-10}$.

Son escasos los hospitales públicos de nuestro país que han adoptado estrategias dirigidas al diagnóstico y tratamiento de los TRS en niños con enfermedades respiratorias crónicas. Por este motivo, el objetivo del presente estudio fue describir los resultados de estudios poligráficos y mostrar su utilidad para el establecimiento de conductas terapéuticas en niños con ENM de un hospital público de Chile.

\section{Pacientes y Métodos}

\section{Diseño}

El diseño de estudio corresponde a una serie retrospectiva de casos, en el cual, los datos fueron registrados por un solo evaluador (RP) desde las fichas clínicas de cada uno de los pacientes.

\section{Pacientes}

Se consideraron todos los registros de poligrafía de niños con ENM pertenecientes al servicio de pediatría del hospital Dr. Guillermo Grant Benavente (HGGB), Concepción, Chile; entre diciembre de 2011 a febrero de 2015. Se excluyeron aquellos pacientes con registros de estudios sin criterios de aceptabilidad; así como también, menores de 3 meses. Por su parte, aquellos pacientes en quienes se realizó más de una PG, sólo fue considerado el primer examen. Puesto que en la totalidad de ellos el segundo examen correspondió a un examen de control que sólo buscó verificar el diagnóstico generado en el primer examen. No existió diferencia significativa entre los resultados de los exámenes diagnósticos y de control (resultados no reportados). El estudio fue aprobado por el comité de ética institucional (Resolución No 2244).

\section{Variables}

Como variables de interés fueron considerados los datos demográficos, diagnóstico, parámetros de poligrafía (duración total de estudio, duración del estudio validado, índice de apnea hipopnea (IAH), índice de apnea hipopnea obstructiva y mixta (IAHOM), número de desaturaciones, saturación mínima y saturación promedio durante el estudio) y determinación de conducta terapéutica; la cual fue determinada luego de la realización, interpretación y análisis de la poligrafía. Como conducta terapéutica fue considerada el plan de intervención posterior al examen, la cual fue categorizada en 3 acciones: Inicio de VNI, tratamiento quirúrgico y observación y seguimiento.

\section{Poligrafía}

En el servicio de pediatría del HGGB fue desarrollado un protocolo de poligrafía nocturna intra- 
hospitalaria, el cual, considera la hospitalización del paciente, así como también, aspectos técnicos y administrativos vinculados a la ejecución del examen. En este contexto, fueron establecidos algoritmos de derivación, hospitalización, responsabilidades de los profesionales y técnicos en cada una de las etapas del examen (Figura 1). El equipo utilizado corresponde a un polígrafo $\mathrm{Ap}$ nea Link Plus (Resmed), de 5 canales: Flujo nasal con transductor nasal de presión, saturación de oxígeno, micrófono, banda torácica y posición; el cual fue operado por una enfermera capacitada en cuanto a los aspectos técnicos y metodológicos del examen ${ }^{11}$.

Durante todo el examen, los niños permanecieron en compañía de sus padres, quienes registraron en un documento ad-hoc (Figura 2): inicio y fin de sueño, despertares, alimentación, controles, vómitos, tos, llanto y pérdida de sensores. Posteriormente el examen es analizado a modo de eliminar los eventos no atribuidos a alteraciones del sueño. El informe final fue generado por un pediatra broncopulmonar de acuerdo a las recomendaciones de Academia Americana de Sueño ${ }^{12}$.

Como criterios de aceptabilidad del examen, fueron considerados: al menos $4 \mathrm{~h}$ de registro, con menos del $20 \%$ del tiempo de registro ocupado por desconexiones y/o artefactos.

La severidad del síndrome de apnea hipopnea de sueño (SAHOS) fue categorizada de acuerdo al valor del índice de apnea/hipopnea (IAH) ${ }^{12}$, en el cual es considerado normal cuando el IAH es $<1$, leve 1-5, moderado 5-10 y severo $>10$.

Los padres firmaron consentimiento informado, así como también, aquellos niños mayores de 12 años firmaron asentimiento informado para la realización de los estudios de sueño.

\section{Análisis estadístico}

En software estadístico MedCalc versión 14.12.0 (MedCalc Software bvba, Ostend, Belgium) se realizó análisis exploratorio de los datos, pruebas de normalidad mediante el test de Shapiro Wilk y estadística descriptiva. Considerando que la muestra no posee distribución normal, estadística no paramétrica fue utilizada. Mediante el test de Kruskal Wallis fue evaluada la diferencia entre los índice de apnea hipopnea entre los grupos de acuerdo a la conducta terapéutica y diagnóstico etiológico. Por otra parte, para evaluar la asociación entre la severidad del TRS (Leve, Moderado y Severo) y la conducta terapéutica fue

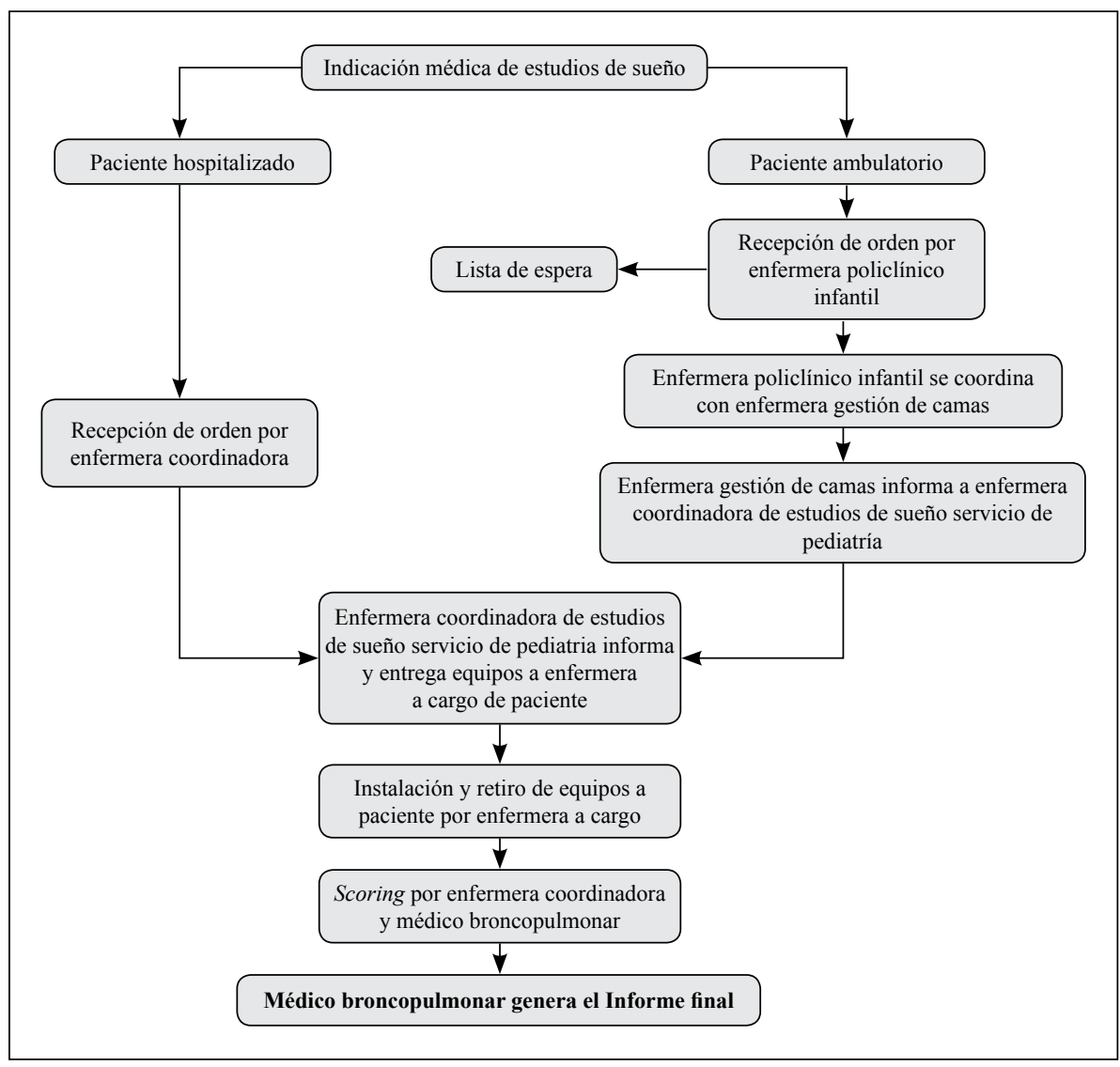

Figura 1. Flujograma de procedimientos de estudios de sueño del hospital Dr. Guillermo Grant Benavente de Concepción. 


\section{FICHA OXIMETRÍA NOCTURNA/POLIGRAFÍA}

\section{I.- DATOS PERSONALES}

\begin{tabular}{|l|l|l|l|l|l|}
\hline Apellido Paterno & \multicolumn{3}{|l|}{ Apellido Materno } & Nombre & \\
\hline $\begin{array}{l}\text { Fecha de } \\
\text { Nacimiento: }\end{array}$ & Edad & $\begin{array}{l}\text { Fecha } \\
\text { Examen }\end{array}$ & RUT & \\
\hline $\begin{array}{l}\text { Nombre Familiar - } \\
\text { Tutor }\end{array}$ & \multicolumn{3}{l|}{} \\
\hline
\end{tabular}

\section{II.- DIAGNÓSTICOS}

\begin{tabular}{|l|l|}
\hline 1 & \\
\hline 2 & \\
\hline 3 & \\
\hline
\end{tabular}

\section{III.- OXÍGENOTERAPIA}

\begin{tabular}{|l|l|l|l|l|l|l|l|}
\hline Equipo & \multicolumn{1}{|l|}{ Fecha inicio } & \multicolumn{3}{|l|}{ Hrs de Uso } & \\
\hline Flujo Indicado & Flujo administrado & & & & & \\
\hline
\end{tabular}

\section{IV.- SATUROMETRÍA NOCTURNA}

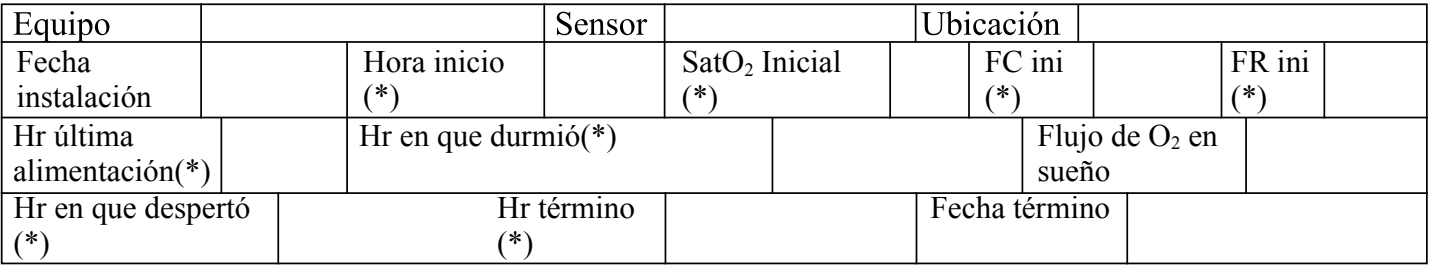

TABLA DE EVENTOS

\begin{tabular}{|c|c|}
\hline \multicolumn{2}{|c|}{ SUEÑO } \\
\hline $\begin{array}{c}\text { Hora } \\
\text { inicio }\end{array}$ & $\begin{array}{c}\text { Hora } \\
\text { término }\end{array}$ \\
\hline & \\
\hline & \\
\hline & \\
\hline & \\
\hline & \\
\hline
\end{tabular}

\begin{tabular}{|c|c|}
\hline \multicolumn{2}{|c|}{ ALIMENTACIÓN } \\
\hline $\begin{array}{c}\text { Hora } \\
\text { inicio }\end{array}$ & $\begin{array}{c}\text { Hora } \\
\text { término }\end{array}$ \\
\hline & \\
\hline & \\
\hline & \\
\hline & \\
\hline & \\
\hline
\end{tabular}

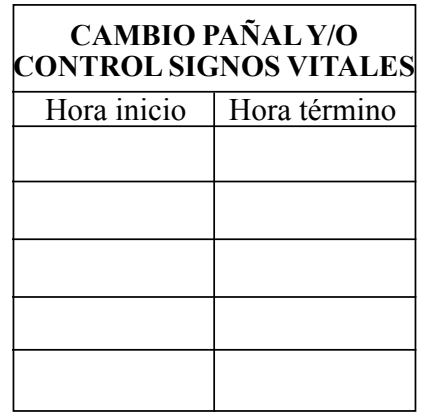

\begin{tabular}{|c|c|}
\hline \multicolumn{2}{|c|}{ MEDICAMENTOS } \\
\hline $\begin{array}{c}\text { Hora } \\
\text { inicio }\end{array}$ & $\begin{array}{c}\text { Hora } \\
\text { termino }\end{array}$ \\
\hline & \\
\hline & \\
\hline & \\
\hline & \\
\hline & \\
\hline
\end{tabular}

\begin{tabular}{|c|c|c|}
\hline \multicolumn{3}{|c|}{ OTROS EVENTOS } \\
\hline $\begin{array}{c}\text { Vómitos, tos, } \\
\text { llanto, etc. }\end{array}$ & $\begin{array}{c}\text { Hora } \\
\text { inicio }\end{array}$ & $\begin{array}{c}\text { Hora } \\
\text { término }\end{array}$ \\
\hline & & \\
\hline & & \\
\hline & & \\
\hline & & \\
\hline & & \\
\hline
\end{tabular}

Figura 2. Documento para registro de eventos durante poligrafía nocturna. 
utilizado el test de Chi-Cuadrado $\left(\chi^{2}\right)$. Un valor de $\mathrm{p}<0,05$ fue considerado significativo.

\section{Resultados}

En el período de tiempo considerado fueron estudiados 36 pacientes. Cinco de ellos fueron excluidos por presentar registro inválido $(\mathrm{n}=3)$ y por tener menos de 3 meses $(n=2)$. Finalmente al estudio ingresaron 31 pacientes.

La mediana de edad fue 10 años (0,3-19), siendo un $74 \%$ de los pacientes de sexo masculino $(\mathrm{n}=23)$. En la Tabla 1 se muestra las características generales de los pacientes estudiados, así como también, la distribución de la muestra de acuerdo a su diagnóstico etiológico.

La mediana de la duración total de los estudios de sueño fue $9,1 \mathrm{~h}(5,3-12,3)$ y el período de tiempo validado 7,3 (4,3-10,5) horas. Por otra parte, la mediana de la saturación promedio durante el estudio fue $97 \%(91 \%-99 \%)$ y la de saturación mínima fue $90 \%(51 \%-95 \%)$. En la Tabla 2 se muestran los resultados de las poligrafías en la muestra total.

El 19\% $(n=6)$ de las PG resultaron normales y $81 \%(n=25)$ fueron sugerentes de trastornos respiratorios del sueño (TRS). Dentro de estas se identificó: SAHOS leve 60\% $(n=15)$, moderado $28 \%(n=7)$ y severo $12 \%(n=3)$. Por su parte, al evaluar la conducta terapéutica en estos pacientes se determinó en $65 \%$ de ellos AVNI $(n=20)$, en $29 \%$ observación y seguimiento $(\mathrm{n}=9)$ y en $7 \%$ tratamiento quirúrgico $(\mathrm{n}=2)$.

No se detectó diferencia estadísticamente significativa en relación al índice de apnea/hipopnea entre los pacientes de acuerdo a su diagnóstico etiológico (Figura 3), no obstante, aquellos pa- cientes con síndrome hipotónico y atrofia espinal presentaron una menor saturación promedio $\mathrm{y}$ saturación mínima (Figura 4 A y B).

No se detectó diferencias estadísticamente

Tabla 1. Características generales de 31 niños con enfermedad neuromuscular

\begin{tabular}{|lc|}
\hline Variable & Mediana (Rango) \\
\hline Sexo (H/M) & $23 / 8$ \\
\hline Edad (años) & $10(0,3-18)$ \\
\hline Diagnóstico & $\mathbf{n}(\%)$ \\
Distrofia muscular de Duchenne & $12(38,7)$ \\
Mielomeningocele & $6(19,4)$ \\
Síndrome hipotónico & $5(16,1)$ \\
Miopatía & $3(9,7)$ \\
Atrofia espinal & $3(9,7)$ \\
Miastenia Gravis & $1(3,2)$ \\
Charcot Marie Tooth & $1(3,2)$ \\
\hline
\end{tabular}

Tabla 2. Resultados de poligrafía respiratoria en 31 niños con enfermedad neuromuscular

\begin{tabular}{|lc|}
\hline Variable & Mediana (Rango) \\
\hline $\begin{array}{l}\text { Duración total PG/ } \\
\text { Tiempo validado (h) }\end{array}$ & $9,1(5,3-12,3) /$ \\
IAH & $7,3(4,3-10,5)$ \\
IAHOM & $2,6(0-19,7)$ \\
Número de desaturaciones & $2,6(0-19,7)$ \\
Saturación mínima (\%) & $16(1-172)$ \\
Saturación promedio (\%) & $90(51-95)$ \\
\hline
\end{tabular}

$\mathrm{PG}=$ Poligrafía; IAH= Índice de apnea hipopnea; IA$\mathrm{HOM}=$ Índice de apnea, hipopnea mixta.

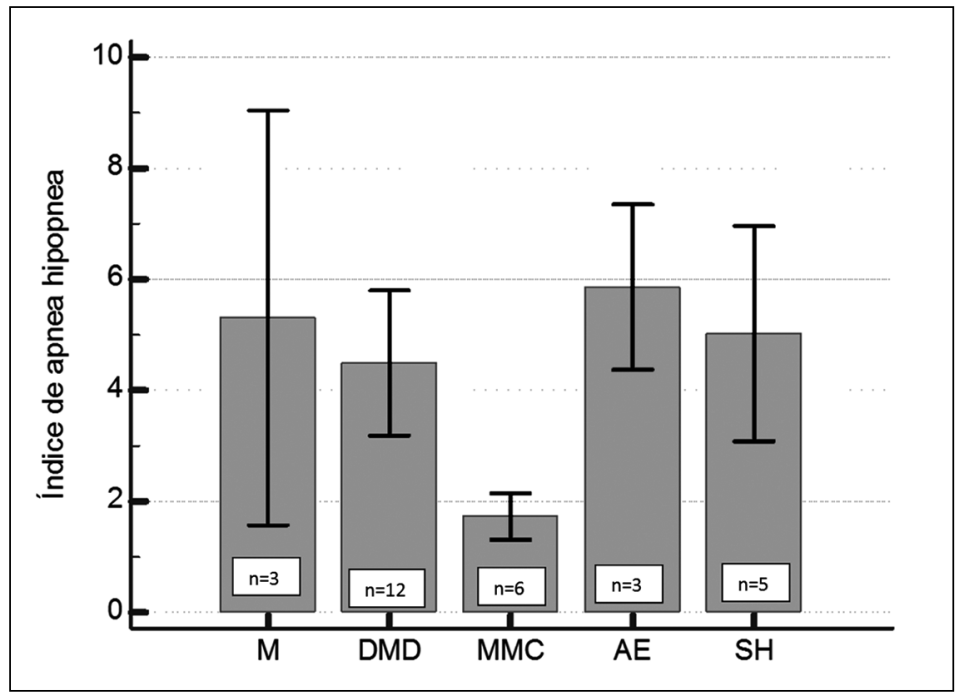

Figura 3. Severidad del SAOS, medido en función del índice de apnea/hipopnea, en cada grupo de pacientes categorizados de acuerdo al diagnóstico etiológico. Resultados se muestran en mediana y error estándar. No existió diferencia significativa entre los grupos. M: Miopatía; DMD: Distrofia muscular de Duchenne; MMC: Mielomeningocele; AE: Atrofia espinal; SH: Síndrome hipotónico. 


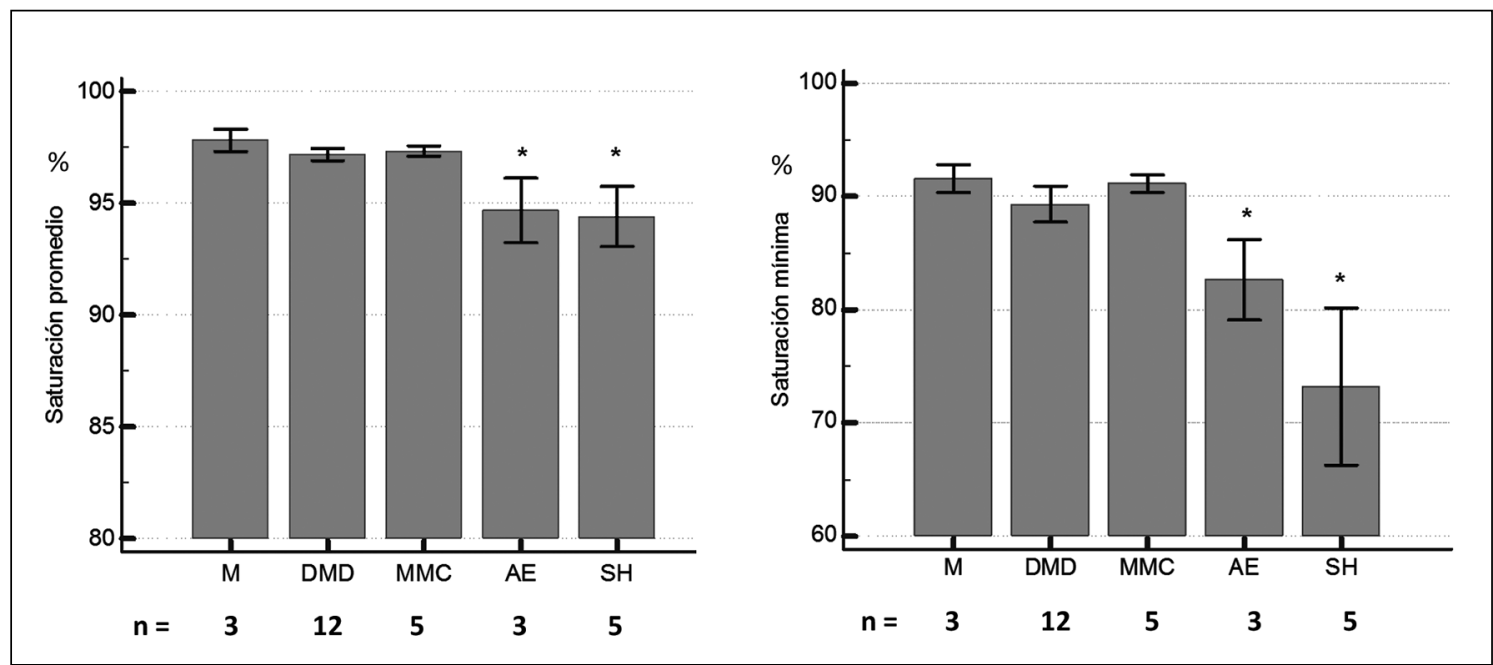

Figura 4. Saturación nocturna en cada grupo de pacientes categorizados de acuerdo al diagnóstico etiológico. Resultados se muestran en mediana y error estándar. En A se muestran resultados de saturación promedio. *Diferencia significativa comparado con los pacientes con diagnóstico de miopatía $(\mathrm{p}<0,05)$. En B se muestran resultados de saturación mínima. *Diferencia significativa comparado con los pacientes con diagnóstico de miopatía, distrofia muscular de Duchenne y mielomeningocele $(\mathrm{p}<0,05)$. M: Miopatía; DMD: Distrofia muscular de Duchenne; MMC: Mielomeningocele; AE: Atrofia espinal; SH: Síndrome hipotónico.

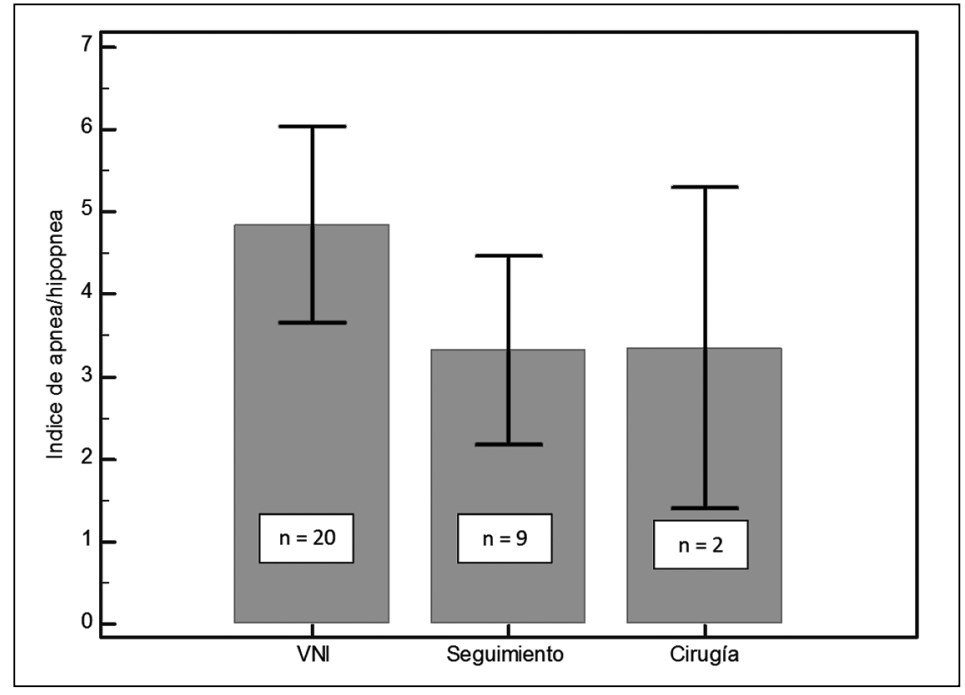

Figura 5. Severidad del SAOS, medido en función del índice de apnea/hipopnea, en cada grupo de pacientes categorizados de acuerdo al tipo de tratamiento recibido. No se detectó diferencia estadística entre el índice de apnea/hipopnea (IAH) y la conducta terapéutica $(\mathrm{p}=0,90)$. Resultados se muestran en mediana y error estándar. VNI: ventilación no invasiva. significativa entre el nivel de índice de apnea/hipopnea de los sujetos categorizados de acuerdo a la conducta terapéutica $(\mathrm{p}=0,90)$ (Figura 5). Así como también, no existió asociación entre el grado de severidad del TRS y la conducta terapéutica de la muestra estudiada $(p=0,75)$.

\section{Discusión}

Mediante este estudio fue posible constatar que el $90 \%$ de los estudios cumplieron con criterios de validez en el primer examen, rendimiento semejante a lo observado en estudios previos tanto en el medio hospitalario como en el domicilio $^{13}$. A su vez, la mediana de edad fue de 10 años, período que se asocia con la declinación de la función respiratoria de los niños con ENM lentamente progresivas ${ }^{4,8-10}$. En este contexto, se ha observado que alrededor de los 6-8 años de vida, los pacientes con ENM experimentan una declinación de su capacidad funcional, comprometiendo el desarrollo motor y la capacidad respiratoria ${ }^{14}$. Por tanto, se ha sugerido que el estudio de los TRS debe preceder el inicio del deterioro funcional en este grupo de pacientes ${ }^{9,15,16}$.

Un 80,6\% de los pacientes estudiados presentó 
algún grado de TRS, la prevalencia de los TRS en niños con ENM no ha sido bien estudiada, no obstante, existen reportes que sugieren que la prevalencia sería mayor que la existente en la población general ${ }^{2,17}$. La prevalencia observada en nuestro estudio puede estar influenciada por la importante heterogeneidad de la muestra y la avanzada edad de los pacientes. Los resultados de las PG permitieron respaldar decisiones terapéuticas en pacientes con ENM. En este ámbito, en el $65 \%$ de los sujetos se inició terapia ventilatoria, lo cual, ha demostrado mitigar el deterioro ventilatorio en este grupo de pacientes ${ }^{17}$. Diversos estudios han observado que, que la atenuación de los TRS prolonga la sobrevida, reduce hospitalizaciones y reagudizaciones respiratorias; así como también, mejora la calidad de vida y disminuye los costos en salud ${ }^{18-22}$.

En aquellos pacientes que tuvieron poligrafías normales $(29 \%)$ se realizó un seguimiento en un policlínico de especialidad del Hospital Guillermo Grant Benavente. Este grupo fue reprogramado para una nueva $\mathrm{PG}$ en un período aproximado de un año según su evolución clínica.

En dos pacientes se determinó tratamiento quirúrgico. En estos casos la indicación quirúrgica se asoció a la presencia concomitante de hipertrofia adenotonsilar grado 3-4.

Algunas debilidades de este estudio corresponden al escaso número de pacientes estudiados, así como también a su heterogeneidad diagnóstica; lo cual, impide la elaboración de conclusiones específicas para cada entidad nosológica. Por otra parte, considerando que no fue utilizada la polisonmografía como método de estudio de sueño, e independientemente al alto rendimiento diagnóstico de la poligrafía; no es posible descartar la existencia de falsos positivos o negativos entre las conclusiones diagnósticas proporcionadas por este instrumento.

\section{Conclusiones}

Finalmente, en virtud de los datos analizados es posible establecer algunas conclusiones. En primer lugar, la PG permitió objetivar la existencia de los TRS en niños con ENM, evaluados en un hospital público de nuestro país. En segundo lugar, el diagnóstico proporcionado por la PG permitió orientar las conductas terapéuticas, especialmente el inicio de VNI.

\section{Agradecimientos}

Queremos agradecer a las enfermeras y auxiliares paramédicos de nuestro Servicio de
Pediatría, Hospital Guillermo Grant Benavente; que participan activamente en la ejecución de los estudios de sueño. A los pacientes y padres que tenemos la fortuna de seguir controlando.

\section{Bibliografía}

1.- GOZAL D. Pulmonary manifestations of neuromuscular disease with special reference to Duchenne muscular dystrophy and spinal muscular atrophy. Pediatr Pulmonol 2000; 29: 141-50.

2.- BROCKMANN V P, HOLMGREN P N, SALINAS M P, PRADO A F. Utilidad de un cuestionario clínico para el diagnóstico de trastornos respiratorios del sueño en niños con enfermedades neuromusculares. Rev Chil Pediatr 2011; 82: 319-28.

3.- SURESH S, WALES P, DAKIN C, HARRIS M A, COOPER D G. Sleep-related breathing disorder in Duchenne muscular dystrophy: disease spectrum in the paediatric population. J Paediatr Child Health 2005; 41: 500-3.

4.- SIMONDS A K. Recent advances in respiratory care for neuromuscular disease. Chest 2006; 130: 1879-86.

5.- MARCUS C L, BROOKS L J, DRAPER K A, GOZAL D, HALBOWER A C, JONES J, et al. Diagnosis and management of childhood obstructive sleep apnea syndrome. Pediatrics 2012; 130: 576-84.

6.- ALONSO ÁlVAREZ M L, TERAN SANTOS J, CORDERO GUEVARA J A, NAVAZO EGUIA A I, ORDAX CARBAJO E, MASA JIMÉNEZ J F, et al. Reliability of respiratory polygraphy for the diagnosis of sleep apneahypopnea syndrome in children. Arch Bronconeumol 2008; 44: 318-23.

7.- ALONSO-ÁlVAREZ M L, TERAN-SANTOS J, ORDAX CARBAJO E, CORDERO-GUEVARA J A, NAVAZO-EGUIA A I, KHEIRANDISH-GOZAL L, et al. Reliability of home respiratory polygraphy for the diagnosis of sleep apnea in children. Chest 2015; 147: 1020-8.

8.- DOHNA-SCHWAKE C, PODLEWSKI P, VOIT T MELLIES U. Non-invasive ventilation reduces respiratory tract infections in children with neuromuscular disorders. Pediatr Pulmonol 2008; 43: 67-71.

9.- FINDER J D, BIRNKRANT D, CARL J, FARBER H J, GOZAL D, IANNACCONE S T, et al. Respiratory care of the patient with Duchenne muscular dystrophy: ATS consensus statement. Am J Respir Crit Care Med 2004; 170: 456-65.

10.- ISHIKAWA Y, MIURA T, ISHIKAWA Y, AOYAGI T, OGATA H, HAMADA S, et al. Duchenne muscular dystrophy: survival by cardio-respiratory interventions. Neuromuscul Disord 2011; 21: 47-51.

11.- RODRÍGUEZ I, NAVARRO X, MORIN M, ZENTENO D. Poligrafía y predictores diurnos de trastornos respiratorios del sueño en niños con enfermedades respiratorias 
crónicas. Rev Cubana Pediatr 2015; 87 (en prensa).

12.- BERRY R B, BUDHIRAJA R, GOTTLIEB D J, GOZAL D, IBER C, KAPUR V K, et al. Rules for scoring respiratory events in sleep: update of the 2007 AASM Manual for the Scoring of Sleep and Associated Events. Deliberations of the Sleep Apnea Definitions Task Force of the American Academy of Sleep Medicine. J Clin Sleep Med 2012; 8: 597-619.

13.- BROCKMANN P, PÉREZ J L MOYA A. Feasibility of unattended home polysomnography in children with sleep-disordered breathing. Int J Pediatr Otorhinolaryngol 2013; 77: 1960-4.

14.- NEVE V, CUISSET J M, EDME J L, CARPENTIER A, HOWSAM M, LECLERC O, et al. Sniff nasal inspiratory pressure in the longitudinal assessment of young Duchenne muscular dystrophy children. Eur Respir J 2013; 42: 671-80.

15.- KATZ S L. Assessment of sleep-disordered breathing in pediatric neuromuscular diseases. Pediatrics 2009; 123 Suppl 4 (S222-5).

16.- PRADO F, SALINAS P, ZENTENO D, VERA R, FLORES E, GARCÍA C, et al. Recomendaciones para los cuidados respiratorios del niño y adolescente con enfermedades neuromusculares. Neumol Pediatr 2010; 5: 74-88.

17.- ZENTENO A D, SALINAS F P, VERA U R, BROCK-
MANN V P, PRADO A F. Enfoque Pediátrico para el Estudio de los Trastornos Respiratorios del Sueño. Rev Chil Pediatr 2010; 81: 445-55.

18.- EAGLE M, BAUDOUIN S V, CHANDLER C, GIDDINGS D R, BULLOCK R, BUSHBY K. Survival in Duchenne muscular dystrophy: improvements in life expectancy since 1967 and the impact of home nocturnal ventilation. Neuromuscul Disord 2002; 12: 926-9.

19.- EAGLE M, BOURKE J, BULLOCK R, GIBSON M, MEHTA J, GIDDINGS D, et al. Managing Duchenne muscular dystrophy-the additive effect of spinal surgery and home nocturnal ventilation in improving survival. Neuromuscul Disord 2007; 17: 470-5.

20.- KIENY P, CHOLLET S, DELALANDE P, LE FORT M, MAGOT A, PEREON Y, et al. Evolution of life expectancy of patients with Duchenne muscular dystrophy at AFM Yolaine de Kepper centre between 1981 and 2011. Ann Phys Rehabil Med 2013; 56: 443-54.

21.- PASSAMANO L, TAGLIA A, PALLADINO A, VIGGIANO E, D'AMBROSIO P, SCUTIFERO M, et al. Improvement of survival in Duchenne Muscular Dystrophy: retrospective analysis of 835 patients. Acta Myol 2012; 31: 121-5.

22.- RALL S, GRIMM T. Survival in Duchenne muscular dystrophy. Acta Myol 2012; 31: 117-20.
Correspondencia a:

Dr. Daniel Zenteno A.

Avenida Alemana n 98, Pedro de Valdivia,

Concepción, Chile.

Email: danielzenteno@gmail.com 